\title{
The Odd Lindley Burr XII Model: Bayesian Analysis, Classical Inference and Characterizations
}

\author{
Mustafa Çăgatay Korkmaz ${ }^{1}$, Haitham M. Yousof ${ }^{2}$, Mahdi Rasekhi ${ }^{3}$ and G. G. Hamedani ${ }^{4}$
}

\begin{abstract}
In this work, we study the odd Lindley Burr XII model initially introduced by Silva et al. [29]. This model has the advantage of being capable of modeling various shapes of aging and failure criteria. Some of its statistical structural properties including ordinary and incomplete moments, quantile and generating function and order statistics are derived. The odd Lindley Burr XII density can be expressed as a simple linear mixture of BurrXII densities. Useful characterizations are presented. The maximum likelihood method is used to estimate the model parameters. Simulation results to assess the performance of the maximum likelihood estimators are discussed. We prove empirically the importance and flexibility of the new model in modeling various types of data. Bayesian estimation is performed by obtaining the posterior marginal distributions as well as using the simulation method of Markov Chain Monte Carlo (MCMC) by the Metropolis-Hastings algorithm in each step of Gibbs algorithm. The trace plots and estimated conditional posterior distributions are also presented.
\end{abstract}

Keywords: Burr XII model; Bayesian estimation; Metropolis-Hastings Algorithm; Multi- censored data; Markov Chain Monte Carlo; Simulation study; Gibbs Algorithm.

\footnotetext{
${ }^{1}$ Corresponding author, Deparment of Measurement and Evaluation, Artvin Ç oruh University, City Campus, Artvin, 08000, Turkey, mcgatay@artvin.edu.tr

2 Department of Statistics, Mathematics and Insurance, Benha University, Benha, Egypt, haitham.yousof@fcom.bu.edu.eg

3 Department of Statistics, Malayer University, Malayer, Iran, rasekhimahdi@gmail.com

${ }^{4}$ Department of Mathematics, Statistics and Computer Science, Marquette University, USA, gholamhoss.hamedani@marquette.edu
} 


\section{Introduction}

Closely resembling the Pearson system of distributions [9, 10], Burr [5] presented a system that incorporates twelve types of cdfs (cumulative distribution function) which yield an assortment of density shapes. This system is obtained by considering cdfs satisfying a differential equation which has a solution, given by $G(y)=\left\{1+\exp \left[-\int \Psi(y) d y\right]\right\}^{-1}$, where $\Psi(y)$ is chosen such that $G(y)$, is a cdf on the real line. Twelve choices for $\Psi(y)$ made by Burr [5], resulted in twelve distributions which might be useful for fitting data. The principal goal in choosing one of these forms of distributions is to facilitate the mathematical analysis to which it is subjected, while attaining a reasonable approximation. Some standard theoretical models are limiting forms of Burr distributions. Burr [5, 6], Hatke [16], Burr and Cislak [7] and Rodriguez [26] paid special attention to one of these forms, Type XII, whose distribution function $G(x)$ is given by

$$
G(x ; \alpha, \beta)=1-\left(1+x^{\alpha}\right)^{-\beta}, x>0,
$$

both $\alpha$ and $\beta$ are positive shape parameters. Location and scale parameters can easily be introduced to make $\mathrm{Eq}(1)$ a four parameter distribution. The cdf and the reliability function of the Burr-XII (BXII) model can be written in a closed form. The corresponding probability density function (pdf) of (1) is given by

$$
g(x ; \alpha, \beta)=\alpha \beta x^{\alpha-1}, x>0 .
$$

The BXII model originally proposed by Burr [5] has many applications in different areas including acceptance sampling plans, reliability and failure time modeling. Tadikamalla [33] studied the BXII model and its related models, namely: Pareto type II (Lomax), log-logistic, compound Weibull gamma and Weibull exponential distributions. Shao et al. [28] extended the three- parameter BXII distribution and used it to model extreme events with applications to flood frequency. Zimmer et al. [38] proposed a new three-parameter Burr XII distribution. This distribution, having Weibull and logistic as sub-models, is a very popular distribution for modeling lifetime data and phenomenon with monotone failure rates. Shao [27] studied the maximum likelihood estimation for the three-parameter BXII model. Soliman [32] studied the estimation of parameters of life from progressively censored data using Burr-XII model. Wu et al. [34] discussed the estimation problems for BXII model on the basis of progressive type II censoring under random removals, where the number of units removed at each failure time has a discrete uniform distribution. Latterly, 
Silva et al. [30] proposed a new location-scale regression model based on BXII model and introduced the log-Burr XII regression models with censored data. Silva et al. [31] proposed a residual for the log-Burr XII regression distribution whose empirical model is close to normal. Korkmaz and Erişoğlu [18] studied on the Burr XII-geometric distribution. Ramos et al. [25] introduced a new five-parameter Burr XII negative binomial model, which has as special cases some important lifetime models discussed in the literature, such as Burr XII, Weibull, the log-logistic and Pareto type II models. Afify at al. [1] studied the Weibull Burr XII distribution. Nasir et al. [20] and Nasir et al. [21] introduced new generalized Burr families of distributions. For the recent extensions of the Burr XII see [3], [8], [35], [36] and [37].

The goal of this article is to study the Odd Lindley-BXII (OLBXII) model first introduced by Silva et al. [29] using the odd Lindley-G (OL-G) family of distributions. The pdf and cdf of the OL-G family of distribution are given by

$$
\mathrm{f}(x ; a, \xi)=\left[a^{2} /(1+a)\right] g(x ; \xi) \bar{G}(x ; \xi)^{-3} \exp [-a G(x ; \xi) / \bar{G}(x ; \xi)], \quad x \in \Re,
$$

and

$$
\mathrm{F}(x ; a, \xi)=1-[a+\bar{G}(x ; \xi)][(1+a) \bar{G}(x ; \xi)]^{-1} \exp [-a G(x ; \xi) / \bar{G}(x ; \xi)], x \in \Re,
$$

respectively. To this end, we use Eq(1)-(3) to obtain the three-parameter OLBXII pdf (for $\mathrm{x}>0)$

$$
f(x ; a, \alpha, \beta)=\left[\frac{a^{2}}{(1+a)}\right] \alpha \beta e^{a} x^{\alpha-1}\left(1+x^{\alpha}\right)^{2 \beta-1} e^{-a\left(1+x^{\alpha}\right)^{\beta}}, x \geq 0
$$

The corresponding cdf of (4) is given by

$$
\mathrm{F}(x ; a, \alpha, \beta)=1-a+\frac{\left(1+x^{\alpha}\right)^{-\beta}}{(1+a)\left(1+x^{\alpha}\right)^{-\beta}} e^{-a\left[\left(1+x^{\alpha}\right)^{\beta}-1\right]}, x \geq 0,
$$

where $\mathrm{a}$ is the positive scale parameter, $\alpha$, and $\beta$ are positive shape parameters. Henceforth, we denote a random variable $X$ having pdf (4) by $\mathrm{X} \sim \operatorname{OLBXII}(a, \alpha, \beta)$. The shapes of the density and hazard rate functions of the OLBXII can also be described analytically. Let

$$
\begin{aligned}
& t_{1}(x)=\frac{\partial}{\partial_{x}}\{\log [f(x)]\}, \text { then } \\
& \quad t_{1}(x)=\frac{\alpha-1}{x}+\frac{\alpha(2 \beta-1) x^{\alpha-1}}{1+x^{\alpha}}-a \alpha \beta x^{\alpha-1}\left(1+x^{\alpha}\right)^{\beta-1}
\end{aligned}
$$

It follows that for $\alpha \leq 1$ and $\beta \leq \frac{1}{2}$, pdf is a decreasing function. Otherwise, the critical point(s) of the OLBXII pdf is (are) the root(s) of 


$$
0=\alpha+\alpha \beta x^{\alpha-1}\left[2 x-a\left(1+x^{\alpha}\right)^{\beta}\right]-x^{\alpha}-1
$$

Now let $t_{2}(x)=\frac{\partial^{2}}{\partial_{x}{ }^{2}}\{\log [f(x)]\}$, then

$$
t_{2}(x)=-\frac{\alpha-1}{x^{2}}+\frac{\alpha x^{\alpha-2}(2 \beta-1)\left(\alpha-1-x^{\alpha}\right)}{\left(1+x^{\alpha}\right)^{2}}-a \alpha \beta x^{\alpha-2}\left(1+x^{\alpha}\right)^{\beta-2}\left(\alpha-1-x^{\alpha}+\alpha \beta x^{\alpha}\right) .
$$

If $x=x_{0}$ is a root of $\operatorname{Eq}(7)$ then it is a local minimum point if $t_{2}(x)>0$, it is a local maximum point if $t_{2}(x)<0$ and it is an inflexion point if $t_{2}(x)=0$. The behavior of the pdf at the end points of the support is given as follows:

$$
\lim _{x \rightarrow 0} f(x)=\left\{\begin{array}{c}
\infty, \alpha<1 \\
\frac{a^{2} \beta}{a+1}, \alpha=1 \\
0, \alpha>1
\end{array},\right.
$$

and

$$
\lim _{x \rightarrow \infty} f(x)=0
$$

The hrf is given by

$$
h(x)=\frac{a^{2} \alpha \beta x^{\alpha-1}\left(1+x^{\alpha}\right)^{2 \beta-1}}{1+a\left(1+x^{\alpha}\right)^{\beta}} .
$$

The critical points of the hrf $h(x)$ are obtained from equation

$$
\frac{\partial}{\partial_{x}}\{\log [h(x)]\}=\frac{\alpha-1}{x}+x^{\alpha-1} \frac{\alpha(2 \beta-1)}{1+x^{\alpha}}-\frac{a \alpha \beta x^{\alpha-1}\left(1+x^{\alpha}\right)^{\beta-1}}{1+a\left(1+x^{\alpha}\right)^{\beta}} .
$$

It follows that for $\alpha \leq 1$ and $\beta \leq 1 / 2$ the hrf is a decreasing function. Otherwise, the critical point(s) of the OLBXII hrf is (are) the $\operatorname{root}(\mathrm{s})$ of the equation

$$
\begin{gathered}
0=(\alpha-1)\left(1+x^{\alpha}\right)\left[1+a\left(1+x^{\alpha}\right)^{\beta}\right]+\alpha(2 \beta-1) x^{\alpha}\left[1+a\left(1+x^{\alpha}\right)^{\beta}\right] \\
-a \alpha \beta x^{\alpha}\left(1+x^{\alpha}\right)^{\beta}
\end{gathered}
$$

The second derivative of $h(x)$, that is $t_{3}(x)=\frac{\partial^{2}}{\partial_{x}{ }^{2}}\{\log [h(x)]\}$, is given by

$$
t_{3}(x)=-\frac{\alpha-1}{x^{2}}+x^{\alpha-1} \frac{\alpha(2 \beta-1)\left(\alpha-1-x^{\alpha}\right) x^{\alpha-2}}{\left(1+x^{\alpha}\right)^{2}}-\frac{\alpha \beta x^{\alpha-2}\left(1+x^{\alpha}\right)^{\beta}+\alpha-1-x^{\alpha}+\alpha \beta x^{\alpha}}{\left[1+a\left(1+x^{\alpha}\right)^{\beta}\right]^{2}} .
$$

If $\mathrm{x}=\mathrm{x}_{0}$ is a root of $\mathrm{Eq}(8)$ then it is a local minimum point if $t_{3}(\mathrm{x})>0$, it is a local maximum point if $t_{3}(x)<0$ and it is an inflexion point if $t_{3}(x)=0$. The behavior of the hrf at the end points of the support is as follows

$$
\lim _{x \rightarrow 0} h(x)=\left\{\begin{array}{c}
\infty, \alpha<1 \\
\frac{a^{2} \beta}{a+1}, \alpha=1 \\
\infty, \alpha<1
\end{array}\right.
$$


and

$$
\lim _{x \rightarrow \infty} h(x)\left\{\begin{array}{c}
0, \alpha \leq 1 \text { and } \beta \leq 0.5 \\
\infty, \text { other cases. }
\end{array} .\right.
$$

Fig. 1 shows that the OLBXII distribution has various pdf shapes such as left-skewed, rightskewed and reversed-J. From Fig. 1 we also see that the shape parameters of the distribution effect its skewness and kurtosis. For example; for fixed a and $\beta$, the kurtosis increases while $\alpha$ increases. Also, same case is valid for fixed $a$ and $\alpha$ while $\alpha$ increases. We will numerically obtain
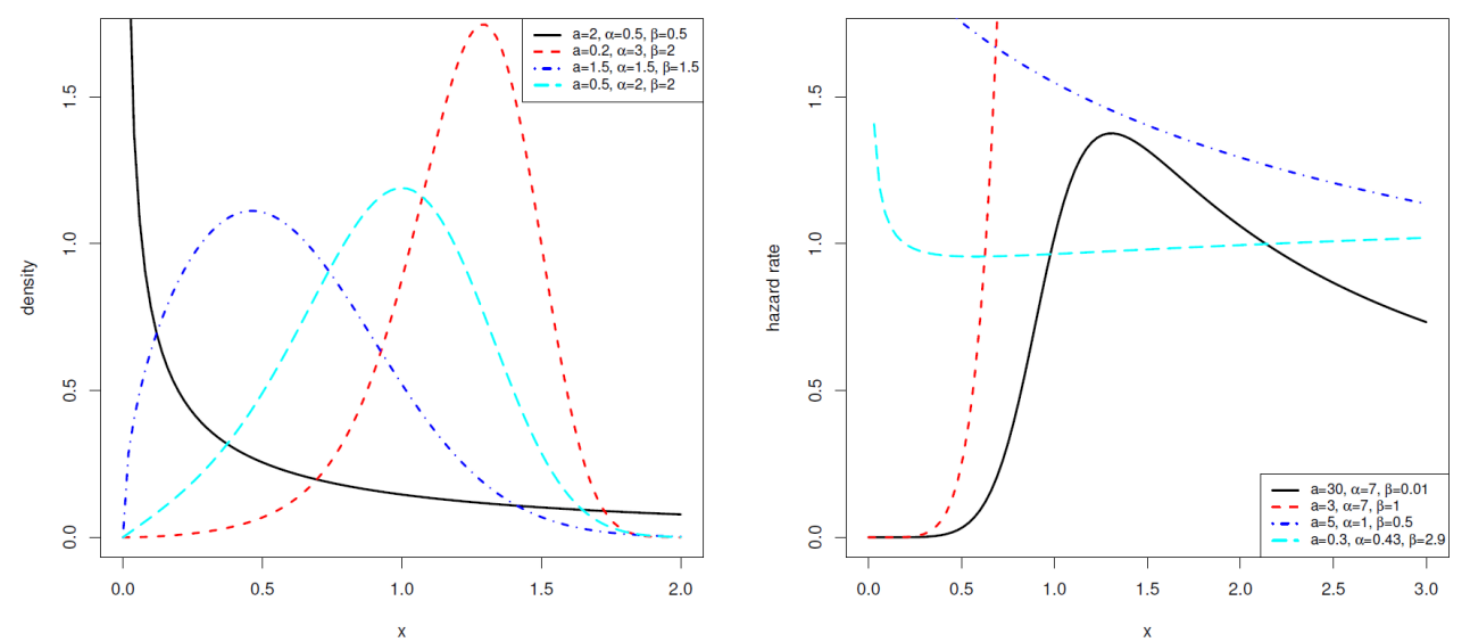

Figure 1: The pdf and hrf of the OLBXII distribution for selected parameter values

the skewness and kurtosis values of the distribution in the next Section. Further, Fig. 2 shows that the OLBXII model produces flexible hazard rate shapes such as increasing, decreasing, bathtub and upsidedown bathtub shaped depending on its selected parameters values. We can say that its shape parameters effective on its different hrf shapes. These plots indicate that the OLBXII model is very useful in fitting different data sets with various shapes.

We give a physical interpretation of OLBXII model as follows. Let $\mathrm{Z}$ be a lifetime random variable having the BXII distribution. The odds ratio that an individual (or component) following the lifetime $\mathrm{Z}$ will die (failure) at time $\mathrm{x}$ is $\frac{1-\left(1+\mathrm{x}^{\alpha}\right)^{-\beta}}{\left(1+\mathrm{x}^{\alpha}\right)^{-\beta}}$. Suppose that the variability of this odds of death is represented by the random variable $\mathrm{X}$ and assume that it follows the Lindley model with scale a. We can write

$$
\operatorname{Pr}(Z \leq x)=\operatorname{Pr}\left(Z \leq \frac{1-\left(1+x^{\alpha}\right)^{-\beta}}{\left(1+x^{\alpha}\right)^{-\beta}}\right)=F(x ; a, \alpha, \beta),
$$


which is given by (6). The pdf in (5) can be expressed as

$$
f(x)=\sum_{r=0}^{\infty} b_{r} g(x ; \alpha, \beta(r+2)) \text {, }
$$

where

$$
b_{r}=\frac{(-1)^{r} \alpha^{r+2} e^{a}}{r !(1+a)(r+2)}
$$

and $\mathrm{g}(x ; a, \alpha, \beta(r+2))$ is the BXII density with parameters $\alpha$ and $\beta(r+2)$, (for more detail about the last two equations see Silva et al. [29]). Similarly,

$$
F(x)=\sum_{r=0}^{\infty} b_{r} G(x ; \alpha, \beta(r+2)),
$$

where $G(x ; \alpha, \beta(r+2))$ is the BXII cdf with parameters $\alpha$ and $\beta(r+2)$. Quantile functions $(\mathrm{QF})$ are in widespread use in statistics and often find representations in terms of lookup tables for key percentiles. The QF say, $x_{u}=Q_{(u)}=F^{-1}(u)$, of $\mathrm{X}$ is a solution of the non-linear equation

$$
0=\mathrm{a}\left[\left(1+x_{u}^{\alpha}-1\right)\right]+\log \left\{\frac{(1-u)(1-a)\left(1-x_{u}^{\alpha}\right)^{-\beta}}{1+\left(1+x_{u}^{\alpha}\right)^{-\beta}}\right\}, 0<u<1 .
$$

Also, these equations can be used for random number generation. Hence, if $U$ is a uniform random variable on $(0,1), X=Q(u)$ follows the OLBXII distribution. From Silva et al. [29], we can write the following equation for QF of the OLBXII distribution

$$
\mathrm{Q}(u)=\left[\left(-a\left[1+W\left((a+1)(u-1) e^{-(a+1)}\right)\right]^{-\frac{1}{\beta}}-1\right)\right]^{\frac{1}{\alpha}}
$$

where $\mathrm{W}(\bullet)$ is Lambert function.

The rest of paper is outlined as follows. In Section 2, we derive some mathematical properties of the new distribution. In Section 3, some characterizations results are provided. In Section 4 we introduce the classical estimation. Bayesian estimation is performed in section 5. In Section 6, we provide the applications to real data sets based on classical approach and Bayesian approach to illustrate the importance of the new distribution. Finally, some concluding remarks are presented in Section 7. 


\section{Properties}

\subsection{General statistical properties}

From $\mathrm{Eq}(9)$, the $n^{\text {th }}$ ordinary moment of $X$ is given by $\mu_{n}^{\prime}=E\left(X^{n}\right)=$ $\sum_{r=0}^{\infty} b_{r} \int_{0}^{\infty} x^{n} g(x ; \alpha, \beta(r+2)) d x$. For $\mathrm{n}<\alpha \beta$, we obtain

$$
\mu_{n}^{\prime}=E\left(X^{n}\right)=\sum_{r=0}^{\infty} b_{r} \beta(r+2) B\left(\beta(r+2)-\frac{n}{\alpha}, \frac{n}{\alpha}+1\right) .
$$

Setting $n=1$ in $\operatorname{Eq}(11)$, we have the mean of $X$. The $s^{\text {th }}$ central moment $\left(M_{s}\right)$ and cumulants $\left(\kappa_{s}\right)$ of $X$, are, respectively, given by $\mathrm{M}_{\mathrm{s}}=E\left(X-\mu_{1}^{\prime}\right)^{s}=$ $\sum_{i=1}^{s}(-1)^{i}\left(\begin{array}{l}s \\ i\end{array}\right)\left(\mu_{1}^{\prime}\right)^{s} \mu_{s-i}^{\prime}$ and $\kappa_{s}=\mu_{s}^{\prime}-\sum_{i=0}^{s-1}\left(\begin{array}{l}s-1 \\ i-1\end{array}\right) \kappa_{r} \mu_{s-r}^{\prime}$ where $\kappa_{1}=\mu_{1}^{\prime}$. The skewness and kurtosis measures can be calculated from the ordinary moments using well-known relationships. We give numerically the moments such as $\mathrm{E}(X)$, variance $\operatorname{Var}(X)$, skewness $\sqrt{\beta_{1}}$ and kurtosis $\beta_{2}$.

From Table 1, we see that the model can be left skewed and right skewed. Also, these results are consistent with Fig 1. The shape parameters effect skewness and kurtosis.

The moment generating function $(\mathrm{mgf})$ of $\mathrm{X}$, say $M_{X}(t)=E[\exp (t X)]$, can be obtained from (9) as $M_{X}(t)=\sum_{r=0}^{\infty} b_{r} M_{r+1}(t)$, where $M_{r+1}(t)$ is the mgf of the BXII distribution with parameters $\alpha, \beta(r+2)$.Paranaiba et al . [22] provided a simple representation for the mgf of the three-parameter BXII distribution. In a similar manner, we provide another representation for the mgf, say $M_{X}(t)$,of the $\operatorname{BXII}(\alpha, \beta)$ model. For $\mathrm{t}<0$, we can write $M_{X}(t)=\alpha \beta \int_{0}^{\infty} \exp (y t) y^{\alpha-1}\left(1+y^{\alpha}\right)^{-\beta-1} d y$. Next, we require the Meijer G-function defined by

$$
G_{p, q}^{m, n}\left(\left.x\right|_{b_{1}} ^{a_{1}}, \cdots, \cdots, \quad \begin{array}{c}
a_{p} \\
b_{q}
\end{array}\right)=\frac{1}{2 \pi i} \int_{L} \frac{\prod_{j=1}^{m} \Gamma\left(b_{j}+t\right) \prod_{j=1}^{m} \Gamma\left(1-a_{j}-t\right)}{\prod_{j=n+1}^{p} \Gamma\left(a_{j}+t\right) \prod_{j=m+1}^{p} \Gamma\left(1-b_{j}-t\right)} x^{-t} d t,
$$

where $\mathrm{i}=\sqrt{-1}$ and $\mathrm{L}$ denotes an integration path (Gradshteyn and Ryzhik [15], Section 9.3).The Meijer G-function includes as particular cases many integrals with elementary and special functions (see [23]). 
Table 1: Some special moments values for selected parameters values of OLBXII

\begin{tabular}{c|cccc}
\hline \hline$a, \alpha, \beta$ & $E(X)$ & $\operatorname{Var}(X)$ & $\sqrt{\beta_{1}}$ & $\beta_{2}$ \\
\hline $0.5,1,0.5$ & 25.3333 & 1416.8890 & 4.2064 & 35.9143 \\
\hline $0.5,1,1$ & 3.3333 & 7.5555 & 1.5123 & 6.3427 \\
\hline $0.5,1,2$ & 0.9890 & 0.3771 & 0.6864 & 3.2654 \\
\hline $1,1,0.5$ & 7.0000 & 99.0000 & 4.0222 & 33.0894 \\
\hline $1,0.5,1$ & 4.0000 & 56.0000 & 5.1161 & 51.5510 \\
\hline $1,1,1$ & 1.5000 & 1.7500 & 1.6198 & 6.7959 \\
\hline $2,0.5,1$ & 2.1666 & 8.1389 & 3.6468 & 27.6014 \\
\hline $0.5,2,0.5$ & 4.1494 & 8.1160 & 1.3910 & 5.9208 \\
\hline $0.5,2,2.5$ & 0.8051 & 0.0724 & -0.2739 & 2.7216 \\
\hline $0.5,2,5$ & 0.5255 & 0.0258 & -0.4907 & 2.9038 \\
\hline $0.5,2,10$ & 0.3577 & 0.0109 & -0.6001 & 3.0430 \\
\hline $2,2,11$ & 0.1905 & 0.0063 & 0.0008 & 2.3929 \\
\hline $0.05,5,2$ & 1.3525 & 0.0183 & -0.8871 & 4.8687 \\
\hline $0.05,5,5$ & 1.0072 & 0.0921 & -0.2019 & 2.9039 \\
\hline $0.05,5,0.5$ & 4.1185 & 1.465143 & 0.2019 & 2.8900 \\
\hline $10,10,10$ & 0.6027 & 0.0049 & -0.7406 & 3.7142 \\
\hline \hline
\end{tabular}

Suppose that $\alpha=m / \beta$, where $m$ and $\beta$ are positive integers. This condition is not restrictive since every positive real number can be approximated by a rational number. Then, we have the following results, which holds for $m$ and $\beta$ positive integers, $\mu>-1$ and $p>0$ (see [24], p. 21),

$$
\begin{aligned}
& I\left(p, \mu, \frac{m}{\beta}, V\right)=\int_{0}^{\infty} \exp (-p x) x^{\mu}\left(1+x^{\frac{m}{\beta}}\right)^{V} d x \\
& =V G_{\beta+m, \beta}^{\beta, \beta+m}\left(\frac{m^{m}}{p^{m}} \mid \begin{array}{c}
\Delta(m,-\mu), \Delta(\beta, V+1) \\
\Delta(\beta, 0)
\end{array}\right)
\end{aligned}
$$

where

$$
V=\beta^{-v} m^{\mu+\frac{1}{2}} /\left[(2 \pi)^{\frac{m-1}{2}} \Gamma(-v) p^{\mu+1}\right],
$$

and

$$
\Delta(\beta, a)=a / \beta,(a+1) / \beta, \cdots,(a+\beta) / \beta .
$$

We can write (for $t<0$ )

$$
M_{X}(t)=m I\left(-t, \frac{m}{\beta}-1, \frac{m}{\beta},-\beta(r+1)\right) .
$$


Hence, the mgf of $\mathrm{X}$ can be expressed as

$$
M_{X}(t)=m \sum_{r=0}^{\infty} b_{r} I\left(-t, \frac{m}{\beta(r+2)}-1, \frac{m}{\beta(r+2)},-\{[\beta(r+2)]+1\}\right) .
$$

The $z^{\text {th }}$ incomplete moment, say $\varphi_{z}(\mathrm{t})=\sum_{r=0}^{\infty} b_{r} \int_{0}^{t} x^{z} g(x ; \alpha, \beta(r+2)) d x$, and then using the lower incomplete gamma function, we obtain(for $z<\alpha \beta$ )

$$
\varphi_{z}(t)=\sum_{r=0}^{\infty} b_{r} \beta(r+2) B\left(t^{\alpha} ; \beta(r+2)-\frac{z}{\alpha}, \frac{z}{\alpha}+1\right) .
$$

The first incomplete moment of $X$, denoted by $\varphi_{1}(t)$, is simply determined from the above equation by setting $z=1$.The first incomplete moment has important applications related to the Bonferroni and Lorenz curves and the mean residual life and the mean waiting time. Furthermore, the amount of scatter in a population is evidently measured to some extent by the totality of deviations from the mean and median. The mean deviations, about the mean and about the median of $X$, depend on $\varphi_{1}(t)$.

\subsection{Order statistics and their moments}

Let $X_{1}, \cdots, X_{n}$ be a random sample of size $\mathrm{n}$ from the OLBXII distribution and let $X_{1: n}, X_{2: n}, \cdots, X_{n: n}$ be the corresponding order statistics.Then, the pdf of the $\mathrm{i}^{\text {th }}$ order statistic $X_{i: n}$, say $\mathrm{f}_{\mathrm{i}: \mathrm{n}}(\mathrm{x})$, is given by

$$
f_{i: n}(x)=\frac{1}{B(i, n-i+1)} f(x) F(x)^{i-1}[1-F(x)]^{n-i} .
$$

By inserting (5) and (6) in equation (12), we obtain

$$
f_{i: n}(x)=\sum_{w=0}^{\infty} t_{w} g(x ; \alpha, \beta(w+1))
$$

where

$$
t_{w}=\sum_{m, p=0}^{\infty} \sum_{j=0}^{k+n-i} \sum_{k=0}^{i-1} \frac{a^{j+m+2}(-1)^{k+m+w}\left(\begin{array}{c}
j+m+p \\
j+m
\end{array}\right)\left(\begin{array}{c}
j+m+p+1 \\
w
\end{array}\right)\left(\begin{array}{c}
k+n-1 \\
j
\end{array}\right)\left(\begin{array}{c}
i-1 \\
k
\end{array}\right)}{\mathrm{B}(i, n-i+1) m !(1+a)^{j+1}(w+1)},
$$

and $g(x ; \alpha, \beta(w+1))$ denotes the BXII density function with parameters $\alpha$ and $\beta(w+1)$. Thus, the density function of the OLBXII order statistics is a linear mixture of two-parameter BXII densities. Based on (13), we can obtain some structural properties of $X_{i: n}$ from those of BXII. The $p^{t h}$ moment of $X_{i: n}$ is given by

$$
E\left(X_{i: n}^{p}\right)=\sum_{w=0}^{\infty} t_{w} \beta(w+1) B\left(\beta(w+1)-\frac{p}{\alpha}, \frac{p}{\alpha}+1\right)
$$

The L-moments are analogous to the ordinary moments and can be estimated by 
employing the linear combinations of the order statistics. Then, using the moments in equation $\mathrm{Eq}(14)$, we can derive explicit expressions for the L-moments of $X$ as infinite weighted linear combinations of the means of suitable OLBXII distributions. They are defined by $(s \geq 1)$

$$
\lambda_{s}=\frac{1}{s} \sum_{d=0}^{z-1}(-1)^{d}\left(\begin{array}{c}
s-1 \\
d
\end{array}\right) E\left(X_{s-d: s}\right), \quad s \geq 1 .
$$

$\mathrm{Eq}(13)$ and (14) are the main result of this subsection.

\section{Characterizations}

This section deals with the characterizations of OLBXII distribution. The first characterization is based on a simple relationship between two truncated moments. It should be mentioned that for this characterization the cdf need not have a closed form. This characterization result employs a theorem due to Glanzel [13], see Theorem 1 of Appendix A. Note that the result holds also when the interval $H$ is not closed. As shown in Glanzel [14], this characterization is stable in the sense of weak convergence. The second characterization is based on the hazard function.

Proposition 1. Let $X: \Omega \rightarrow(0, \infty)$ be a continuous random variable and let $q_{1}(x)=$ $\left(1+x^{\alpha}\right)^{-\beta}$ and $q_{2}(x)=q_{1}(x)^{-a\left(1+x^{\alpha}\right)^{\beta}}$ for $x>0$. The random variable $X$ belongs to OLBXII family (5) if and only if the function $\eta$ defined in Theorem 1 has the form

$$
\eta(x)=\frac{1}{2} e^{-a\left(1+x^{\alpha}\right)^{\beta}}, x>0
$$

Proof. Let $\mathrm{X}$ be a random variable with pdf $\mathrm{Eq}(5)$, then

$$
(1-F(x)) E\left[q_{1}(x) \mid X \geq x\right]=\frac{a e^{a}}{1+a} e^{-a\left(1+x^{\alpha}\right)^{\beta}}, x>0,
$$

and

$$
(1-F(x)) E\left[q_{2}(x) \mid X \geq x\right]=\frac{a e^{a}}{2(1+a)} e^{-2 a\left(1+x^{\alpha}\right)^{\beta}}, x>0,
$$

and finally

$$
\eta(x) q_{1}(x)-q_{2}(x)=-\frac{1}{2} q_{1}(x) e^{-a\left(1+x^{\alpha}\right)^{\beta}}<0 \quad \text { for } \quad x>0 .
$$


Conversely, if $\eta$ is given as above, then

$$
s^{\prime}(x)=\frac{\eta^{\prime}(x) q_{1}(x)}{\eta(x) q_{1}(x)-q_{2}(x)}=a \alpha \beta x^{\alpha-1}\left(1+\mathrm{x}^{\alpha}\right)^{\beta-1}, \quad x>0,
$$

and hence

$$
s(x)=a\left(1+\mathrm{x}^{\alpha}\right)^{\beta}, x>0 .
$$

Now, in view of Theorem1, $\mathrm{X}$ has density (5).

Remark 1. The condition $\mathbf{E}\left[q_{2}(x) \mid X \geq x\right]=\mathbf{E}\left[q_{1}(x) \mid X \geq x\right] \eta(x), x \in H$, of Theorem 1 can be replaced with $\mathbf{E}\left[q_{2}(x) \mid X \geq x\right]=\eta(x), \mathrm{x} \in \mathrm{H}$, by taking $q_{1}(x)=1$. This, however, limits the domain of applicability of this theorem. That being said, in some cases the latter condition is more appropriate.

Corollary 1. Let $X: \Omega \rightarrow(0, \infty)$ be a continuous random variable and let $q_{1}(x)$ be as in Proposition 1. The pdf of $X$ is $\mathrm{Eq}(5)$ if and only if there exist functions $\mathrm{q}_{2}$ and $\eta$ defined in Theorem1 satisfying the differential equation

$$
\frac{\eta^{\prime}(x) q_{1}(x)}{\eta(x) q_{1}(x)-q_{2}(x)}=a \alpha \beta x^{\alpha-1}\left(1+x^{\alpha}\right)^{\beta-1}, \quad x>0 .
$$

The general solution of the differential equation in Corollary 1 is

$$
\eta(x)=e^{a\left(1+\mathrm{x}^{\alpha}\right)^{\beta}}\left[-\int a \alpha \beta x^{\alpha-1}\left(1+x^{\alpha}\right)^{\beta-1} e^{-a\left(1+\mathrm{x}^{\alpha}\right)^{\beta}}\left(q_{1}(x)\right)^{-1} q_{2}(x) d x+D,\right],
$$

where $D$ is a constant. Note that a set of functions satisfying the above differential equation is given in Proposition 1 with $=\frac{1}{2}$. However, it should be also mentioned that there are other triplets $\left(q_{1}, q_{2}, \eta\right)$ satisfying the conditions of Theorem1.

It is known that the hazard function, $h_{F}$, of a twice differentiable distribution function, $F$, satisfies the first order differential equation

$$
\frac{f^{\prime(x)}}{f^{\prime(x)}}=\frac{h_{F}^{\prime}(x)}{h_{F}(x)}-h_{F}(x) .
$$

For many univariate continuous distributions, this is the only characterization available in terms of the hazard function. The following proposition establish a non-trivial characterization of OLBXII distribution, in terms of the hazard function, which is not of the above trivial form given.

Proposition 2. Let $X: \Omega \rightarrow(0, \infty)$ be a continuous random variable. The pdf of $X$ is $\mathrm{Eq}(5)$ if and only if its hazard function $h_{F}(x)$ satisfies the differential equation 


$$
h_{F}^{\prime}(x)-(\alpha-1) x^{-1} h_{F}(x)=a^{2} \alpha \beta x^{\alpha-1} \frac{d}{d x}\left\{\frac{\left(1+x^{\alpha}\right)^{2 \beta-1}}{a+\left(1+x^{\alpha}\right)^{-\beta}}\right\}
$$

or

$$
h_{F}(x)=\frac{a^{2} \alpha \beta x^{\alpha-1}\left(1+x^{\alpha}\right)^{2 \beta-1}}{a+\left(1+x^{\alpha}\right)^{-\beta}}, x>0,
$$

which is the hazard function of the OLBXII distribution.

\section{Classical inference}

In subsection 4.1, we introduce a procedure for maximum likelihood estimation of the parameters of the OLBXII model. Subsection 4.2 assesses the performance of the maximum likelihood estimators (MLEs) by means of a simulation study.

\subsection{Maximum likelihood estimation}

We consider the estimation of the unknown parameters of the OLBXII model from complete samples only by maximum likelihood method. The MLEs of the parameters of the OLBXII $(a, \alpha, \beta)$ model is now discussed. Let $x_{1}, \cdots, x_{n}$ be a random sample of this distribution with parameter vector $\Theta=(a, \alpha, \beta)^{\top}$;. The log-likelihood function for $\theta$, say $\ell=\ell(\Theta)$, is given by

$$
\begin{aligned}
\ell=\ell(\Theta)=2 n & \log (a) \\
& +n \log (\alpha)+n \log (\beta) \\
& -n \log (1+a)+(\alpha-1) \sum_{i=1}^{n} \log x_{i}+(2 \beta \\
& -1) \sum_{i=1}^{n} \log \left(1+x_{i}^{\alpha}\right)+\sum_{i=1}^{n}\left\{-a\left[\left(1+x^{\alpha}\right)^{\beta}-1\right]\right\} .
\end{aligned}
$$

The last equation can be maximized either by using different programs like $\mathrm{R}$ (optim function),SAS (PROC NLMIXED) or by solving the nonlinear likelihood equations obtained by differentiating Eq(15). The score vector elements, $U(\Theta)=\frac{\partial \ell}{\partial \theta}=\left(\frac{\partial \ell}{\partial a}, \frac{\partial \ell}{\partial \alpha}, \frac{\partial \ell}{\partial \beta}\right)^{\top}$, are given in Appendix B. We can obtain the estimates of the unknown parameters by setting the score vector to zero, $U(\widehat{\Theta})=0$. Solving these equations simultaneously gives the MLEs $\hat{a}, \hat{\alpha}$ and $\hat{\beta}$. For the OLBXII distribution, all the second order derivatives exist. The interval estimation of the model parameters requires the $3 \times 3$ observed information 
matrix $J(\Theta)=\left\{J_{i j}\right\}$ for $i, j=a, \alpha, \beta$. The multivariate normal $N_{3}\left(0, J(\widehat{\Theta})^{-1}\right)$ distribution, under standard regularity conditions, can be used to provide approximate confidence intervals for the unknown parameters, where $J(\widehat{\Theta})$ is the total observed information matrix evaluated at $\hat{\Theta}$. Then, approximate $100(1-\delta) \%$ confidence intervals for $a, \alpha$ and $\beta$ can be determined by:

$\hat{a} \pm z_{\delta / 2} \sqrt{\hat{\jmath}_{a \alpha}}, \hat{\alpha} \pm z_{\delta / 2} \sqrt{\hat{\jmath}_{a \alpha}}$ and $\hat{\beta} \pm z_{\delta / 2} \sqrt{\hat{\jmath}_{\beta \beta}}$ where $z_{\delta / 2}$ is the upper $\delta^{\text {th }}$ percentile of the standard normal model.

\subsection{Simulation Study}

To see the performance of the maximum likelihood estimates, we generate 1,000 samples of sizes 50,100 and 200 from the $\operatorname{OLBXII}(a, \alpha, \beta)$ distribution. The results of the simulation are reported in Table 2 . We observe that the estimates approach true values as the sample size increases, implying the consistency of the estimators. Also, we observe, from Table 2, that the estimators are quite stable and get closer to the true values as the sample sizes increases.

Table 2: Empirical means and standard deviations (given in parentheses)

for different values of the OLBXII parameters.

\begin{tabular}{|c|c|c|c|c|c|c|c|c|c|}
\hline Parameters & & $\mathrm{n}=50$ & & & $\mathrm{n}=100$ & & & $\mathrm{n}=200$ & \\
\hline$a, \alpha, \beta$ & $\hat{a}$ & $\hat{\alpha}$ & $\hat{\beta}$ & $\hat{a}$ & $\hat{\alpha}$ & $\hat{\beta}$ & $\hat{a}$ & $\hat{\alpha}$ & $\hat{\beta}$ \\
\hline \multirow[t]{2}{*}{$0.5,1,0.5$} & 0.4895 & 1.3408 & 0.5558 & 0.5075 & 1.0943 & 0.5343 & 0.4965 & 1.0348 & 0.5210 \\
\hline & $(0.2255)$ & (1.3223) & $(0.4057)$ & $(0.1617)$ & $(0.4043)$ & $(0.2470)$ & $(0.1042)$ & $(0.2324)$ & $(0.1591)$ \\
\hline \multirow[t]{2}{*}{$1,1,0.5$} & 1.1411 & 1.0379 & 0.5957 & 0.9471 & 1.0584 & 0.5743 & 1.0327 & 1.0380 & 0.5023 \\
\hline & $(1.1268)$ & $(0.2999)$ & $(0.3580)$ & $(0.3327)$ & $(0.2147)$ & $(0.2330)$ & $(0.2487)$ & $(0.1469)$ & $(0.1378)$ \\
\hline \multirow[t]{2}{*}{$0.5,0.5,1$} & 0.5536 & 0.5426 & 1.1543 & 0.5464 & 0.5307 & 1.0230 & 0.4852 & 0.4936 & 1.0105 \\
\hline & $(0.3093)$ & $(0.2257)$ & $(0.5526)$ & $(0.2027)$ & $(0.1361)$ & $(0.3995)$ & $(0.1555)$ & $(0.1323)$ & $(0.3462)$ \\
\hline \multirow[t]{2}{*}{$1,1,1$} & 1.1352 & 1.0505 & 1.1770 & 1.0511 & 0.9720 & 1.1689 & 1.0329 & 0.9877 & 1.1639 \\
\hline & $(0.8053)$ & $(0.3173)$ & $(0.8296)$ & $(0.5975)$ & $(0.2391)$ & $(0.7183)$ & $(0.4570)$ & $(0.2141)$ & $(0.6576)$ \\
\hline \multirow[t]{2}{*}{$2,2,1$} & 2.7232 & 1.9897 & 1.3704 & 2.2061 & 1.9766 & 1.1525 & 2.1048 & 2.0089 & 1.0221 \\
\hline & $(5.4551)$ & $(0.4939)$ & $(0.8492)$ & $(1.2370)$ & $(0.3561)$ & $(0.6187)$ & $(1.2042)$ & $(0.2761)$ & $(0.3799)$ \\
\hline
\end{tabular}




\begin{tabular}{|c|c|c|c|c|c|c|c|c|c|}
\hline \multirow{2}{*}{$\begin{array}{c}\text { Parameters } \\
a, \alpha, \beta\end{array}$} & \multicolumn{2}{|c|}{$\mathrm{n}=50$} & \multicolumn{4}{|c|}{$\mathrm{n}=100$} & \multicolumn{3}{|c|}{$\mathrm{n}=200$} \\
\hline & $\hat{a}$ & $\hat{\alpha}$ & $\hat{\beta}$ & $\hat{a}$ & $\hat{\alpha}$ & $\hat{\beta}$ & $\hat{a}$ & $\hat{\alpha}$ & $\hat{\beta}$ \\
\hline \multirow[t]{2}{*}{$1,1,2$} & 1.4087 & 1.0598 & 2.1014 & 1.3770 & 1.0297 & 2.0538 & 1.2795 & 1.0343 & 2.0291 \\
\hline & 2.2545) & $(0.3292)$ & (1.1300) & $(1.0820)$ & $(0.2848)$ & $(1.0529)$ & $(0.8505)$ & $(0.2224)$ & $(0.8014)$ \\
\hline \multirow[t]{2}{*}{$2,1,1$} & 2.9015 & 0.9626 & 1.3016 & 2.5855 & 1.0161 & 1.0829 & 2.4898 & 0.9974 & 1.0390 \\
\hline & $(4.8353)$ & $(0.2782)$ & $(0.9465)$ & (2.7291) & $(0.2136)$ & $(0.7615)$ & (1.5767) & $(0.1454)$ & $(0.4689)$ \\
\hline \multirow[t]{2}{*}{$1,2,2$} & 1.8506 & 2.1086 & 2.0742 & 1.3295 & 2.0601 & 2.0477 & 1.2236 & 2.0136 & 2.0559 \\
\hline & $(3.3035)$ & $(0.5680)$ & $(1.0002)$ & (1.0219) & $(0.5114)$ & $(0.9913)$ & $(0.8246)$ & $(0.4305)$ & $(0.8213)$ \\
\hline \multirow[t]{2}{*}{$1,2,1$} & 1.3858 & 2.0978 & 1.1575 & 1.0620 & 1.9818 & 1.1472 & 1.0221 & 1.9906 & 1.1293 \\
\hline & (1.1533) & $(0.6109)$ & $(0.7697)$ & $(0.4878)$ & $(0.4128)$ & $(0.6558)$ & $(0.4043)$ & $(0.3569)$ & $(0.6097)$ \\
\hline \multirow[t]{2}{*}{$1,2,0.5$} & 1.0383 & 2.2615 & 0.5417 & 1.0081 & 1.9997 & 0.5378 & 0.9988 & 2.0041 & 0.5023 \\
\hline & $(0.4813)$ & $(0.7124)$ & $(0.2973)$ & $(0.3218)$ & $(0.4426)$ & $(0.1854)$ & $(0.2535)$ & $(0.3108)$ & $(0.1451)$ \\
\hline \multirow[t]{2}{*}{$0.5,2,1$} & 0.5302 & 2.1699 & 1.1839 & 0.5226 & 2.0983 & 1.1693 & 0.4854 & 1.9920 & 1.1545 \\
\hline & $(0.3391)$ & $(1.0044)$ & $(0.6191)$ & $(0.2556)$ & $(0.6931)$ & $(0.5723)$ & $(0.1858)$ & $(0.5425)$ & $(0.5439)$ \\
\hline \multirow[t]{2}{*}{$3,2,1$} & 4.0529 & 1.9193 & 1.2982 & 3.1181 & 1.9614 & 1.1660 & 2.9511 & 1.9728 & 1.0753 \\
\hline & $(6.1738)$ & $(0.4424)$ & (1.0506) & (5.4403) & $(0.3451)$ & $(0.8699)$ & $(4.2879)$ & $(0.2430)$ & $(0.5721)$ \\
\hline \multirow[t]{2}{*}{$1,2,3$} & 1.4683 & 2.2275 & 2.7639 & 1.2230 & 2.0817 & 3.0944 & 1.0225 & 1.9654 & 3.0469 \\
\hline & $(4.2676)$ & $(0.6452)$ & $(1.2344)$ & $(2.0516)$ & $(0.5492)$ & (1.1112) & $(0.6664)$ & $(0.4567)$ & (1.1519) \\
\hline
\end{tabular}

\section{Bayesian Estimation}

The goal of this section is to introduce a way to generate data from joint posterior distribution of OLBXII parameters. A regular procedure is the simulation method of MCMC by the Metropolis- Hastings algorithm in each step of Gibbs algorithm. Since prior information about parameters in the history of data does not exist, weakly informative prior distributions for the parameters $a, \alpha$ and $\beta$ are selected. Also, independance assumption is considered for the elements of the parameter vector. Thus, the joint prior distribution of the unknown parameters will have the following pdf

$$
\pi(\mathrm{a}, \alpha, \beta)=\pi(\mathrm{a},) \times \pi(\alpha) \times \pi(\beta)=\Gamma\left(c_{1}, b_{1}\right) \times \Gamma\left(c_{2}, b_{2}\right) \times \Gamma\left(c_{3}, b_{3}\right),
$$


where $\Gamma\left(c_{i}, b_{i}\right)$ denotes a gamma distribution with mean $\frac{c_{i}}{b_{i}}$ and variance $\frac{c_{i}}{b_{i}^{2}}$. All hyper-parameters are known. The joint posterior distribution for parameters $(a, \alpha, \beta)$ is obtained by the product of the likelihood function (15) and the prior distribution (16) as follow

$$
\begin{aligned}
\pi(\mathrm{a}, \alpha, \beta \mid x)= & \left.\frac{a^{2} \alpha \beta}{1+a}\right)^{n}\left(\prod_{i=1}^{n} x_{i}\right)^{\alpha-1}\left(\prod_{i=1}^{n}\left(1+x_{i}^{\alpha}\right)\right)^{2 \beta-1} \\
& \times \exp \left(-a \sum_{i=1}^{n}\left[\left(1+x_{i}^{\alpha}\right)^{\beta}-1\right]\right) \pi(\mathrm{a}, \alpha, \beta)
\end{aligned}
$$

The integration of the joint posterior density is not easy since, the joint posterior density is analytically intractable. Thus, the full conditional distributions of the unknown parameters are given by

$$
\begin{aligned}
& \pi(a \mid, x, \alpha, \beta) \propto \frac{a^{2 n+c_{1}-1}}{(1+a)^{n}} \exp \left(-a\left\{\left(\sum_{i=1}^{n}\left[\left(1+x_{i}^{\alpha}\right)^{\beta}-1\right]\right)+b_{1}\right\}\right), \\
& \pi(\alpha \mid, x, a, \beta) \propto \alpha^{n+c_{2}-1} \exp \left(-\alpha\left(b_{2}-\sum_{i=1}^{n} \log \left(x_{i}\right)\right)\right) \\
& \times\left(\prod_{i=1}^{n}\left(1+x_{i}^{\alpha}\right)\right)^{2 \beta-1} \exp \left(-a \sum_{i=1}^{n}\left[\left(1+x_{i}^{\alpha}\right)^{\beta}-1\right]\right),
\end{aligned}
$$

and

$$
\begin{aligned}
\pi(\beta \mid, x, a, \alpha) \propto & \beta^{n+c_{3}-1} \exp \left(-\beta\left(b_{3}-2 \sum_{i=1}^{n} \log \left(1+x_{i}^{\alpha}\right)\right)\right) \\
& \times \exp \left(-a \sum_{i=1}^{n}\left[\left(1+x_{i}^{\alpha}\right)^{\beta}-1\right]\right) .
\end{aligned}
$$

Since the full conditional distributions for $(a, \alpha, \beta)$ do not have explicit expressions, the Metropolis-Hastings algorithm is required in each step of Gibbs algorithm. 


\section{Applications}

\subsection{Using classical approach}

In this subsection, we show the fitting of the OLBXII distribution to two real uncensored data sets. For these data sets, we also compare the OLBXII distribution with BXII distribution and Marshall-Olkin Burr XII (MOBXII) distribution [2] under $\hat{\ell}$ value, Akaike information criteria (AIC), Bayesian information criteria (BIC), Kolmogorov-Smirnov (K-S), Anderson-Darling $\left(A^{*}\right)$ and Cramer-Von Moses $\left(W^{*}\right)$ goodness-of-fit statistics. The corresponding density of MOBXII model is $f_{\text {MOBXII }}(x)=a \alpha \beta x^{\alpha-1}\left(1+x^{\alpha}\right)^{\beta-1}[1-$ $\left.(1-a)\left(1+x^{\alpha}\right)^{-\beta}\right]^{-2}$ for $x, \mathrm{a}, \alpha, \beta>0$. All the computations were performed by using the maxLik procedure in $\mathrm{R}$ statistical software.

The first real data set gives the survival times, in weeks, of 33 patients suffering from acute Myelogeneous Leukaemia. These data have been analyzed by Feigl and Zelen [11] and Mead [19]. The data are: 65, 156, 100, 134, 16, 108, 121, 4, 39, 143, 56, 26, 22, 1, 1, 5, $65,56,65,17,7,16,22,3,4,2,3,8,4,3,30,4,43$. Table 2 lists the results of this data analysis.

Based on the values in Table 3, the OLBXII model provides adequate fits as compared to MOBXII and BXII models in application with small values for AIC, BIC, $A^{*}, W^{*}$ statistics and big value for $\hat{\ell}$. Based on the data set, the new OLBXII model is much better than the MOBXII and BXII models.

The estimated variance-covariance matrix (inverse of the observed Fisher information matrix) is given by

$$
J(\widehat{\Theta})^{-1}=\left(\begin{array}{ccc}
0.0625 & 0.5960 & -0.0120 \\
0.5960 & 139.3457 & -1.9558 \\
-0.0120 & -1.9558 & 0.0277
\end{array}\right) .
$$


Table 3 : MLEs, $\hat{\ell}$ values (standard errors in parentheses) for OLBXII, MOBXII and BXII models and the statistics AIC, BIC, K-S, $A^{*}$ and $W^{*}$ based on Myelogeneous Leukaemia data $([\cdot]\{\cdot\}$ and $(\cdot)$ denote their p-values $)$

\begin{tabular}{|c|c|c|c|c|c|c|c|c|c|}
\hline Model & $\hat{a}$ & $\hat{\alpha}$ & $\hat{\beta}$ & $\hat{\ell}$ & $A I C$ & $B I C$ & $K-S$ & $A^{*}$ & $W^{*}$ \\
\hline \multirow[t]{2}{*}{ OLBXII } & 0.6237 & 5.3979 & 0.0743 & -149.7538 & 305.5075 & 309.9970 & 0.1294 & 0.5889 & 0.0885 \\
\hline & $(0.2500)$ & (11.8044) & $(0.1664)$ & & & & [0.6384] & $\{0.6572\}$ & $(0.6476)$ \\
\hline \multirow[t]{2}{*}{ MOBXI } & 11.2992 & 4.6824 & 0.1996 & -151.7895 & 309.5791 & 310.4066 & 0.1270 & 0.6765 & 0.1001 \\
\hline & (6.4851) & (4.4505) & $(0.1965)$ & & & & [0.6623] & $\{0.5775\}$ & $(0.5869)$ \\
\hline BXII & & 25.7207 & 0.0141 & -158.6288 & 321.2576 & 324.2506 & 0.2376 & 2.6918 & 0.4938 \\
\hline
\end{tabular}

Hence, the $90 \%$ confidence intervals for $a, \alpha$ and $\beta$ parameters are given by [0.2125,1.0349], [ $-7.6120,31.2209]$ and [ $-0.1995,0.3481]$ respectively.

To illustrate the performance of our newly proposed distribution further, we introduce another example of Average Annual Percent Change in Private Health Insurance Premiums (All Benefits: Health Services and Supplies), Calendar Years 1969-2007 (SOURCE: Centers for Medi- care \& Medicaid Services, Office of the Actuary, National Health Statistics Group). These data have been analyzed by Kibria and Shakil [17]. The data are:14.4, 14.0, 15.4, 9.4, 11.7, 15.0, 24.9, 20.7, 12.5, 14.9, 12.6, 16.7, 13.8, 11.0, 12.9, 10.1, $1.9,8.5,16.5,15.3,13.3,9.8,8.4,7.9,3.7,5.1,4.6,4.4,5.4,6.1,8.0,10.0,11.2,10.1,6.4$, $6.7,5.7,5.8$. Table 3 lists the results of this data analysis.

Table 4 : MLEs, $\hat{\ell}$ values (standard errors in parentheses) for OLBXII, MOBXII and BXII models and the statistics AIC, BIC, K-S, $A^{*}$ and $W^{*}$ based on Average Annual Percent $([\cdot]\{\cdot\}$ and $(\cdot)$ denote their p-values $)$

\begin{tabular}{|c|c|c|c|c|c|c|c|c|c|}
\hline Model & $\hat{a}$ & $\hat{\alpha}$ & $\hat{\beta}$ & $\hat{\ell}$ & $A I C$ & $B I C$ & $K-S$ & $A^{*}$ & $W^{*}$ \\
\hline \multirow[t]{2}{*}{ OLBXII } & 0.0469 & 11.3551 & 0.1366 & -112.5248 & 231.0497 & 235.9624 & 0.0762 & 0.2366 & 0.0363 \\
\hline & $(0.0238)$ & (3.0446) & $(0.0403)$ & & & & {$[0.9801]$} & $\{0.9769\}$ & $(0.9531)$ \\
\hline \multirow[t]{2}{*}{ MOBXI } & 137.1441 & 220.7813 & 0.0103 & -118.4854 & 242.97083 & 247.8836 & 0.1494 & 1.6766 & 0.2800 \\
\hline & $(8.6207)$ & $(2.9062)$ & $(0.0005)$ & & & & [0.3634] & f0.1396g & $(0.1540)$ \\
\hline \multirow[t]{2}{*}{ BXII } & & 10.6930 & 0.0416 & -154.0969 & 312.1938 & 315.4689 & 0.4299 & 10.3760 & 2.1648 \\
\hline & & $(5.1426)$ & $(0.0211)$ & & & & {$[0.000002]$} & $\mathrm{f} 0.00002 \mathrm{~g}$ & $(0.000003)$ \\
\hline
\end{tabular}


Based on the values in Table 4, the OLBXII model provides adequate fits as compared to MOBXII and BXII models in application with small values for AIC, BIC and with big values for log-likelihood. Based on the data set, the new OLBXII model is much better than the MOBXII and BXII models.

The estimated variance-covariance matrix is given by

$$
J(\widehat{\Theta})^{-1}=\left(\begin{array}{ccc}
0.0006 & 0.00003 & -0.0004 \\
0.0003 & 9.2675 & -0.1115 \\
-0.0004 & -0.1115 & 0.0016
\end{array}\right) .
$$

Hence, the $90 \%$ confidence intervals for $a, \alpha$ and $\beta$ parameters are given by $[0.0066$, 0.0872], [6.3477, 16.3625] and [0.0708, 0.2024] respectively.

Figure 2 and Figure 3 display the probability-probability (P-P) plots for the application models. It is evident from these plots that the OLBXII distribution provides superior fit to both data set.

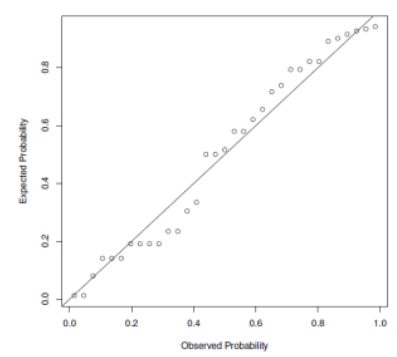

(a) OLBXII

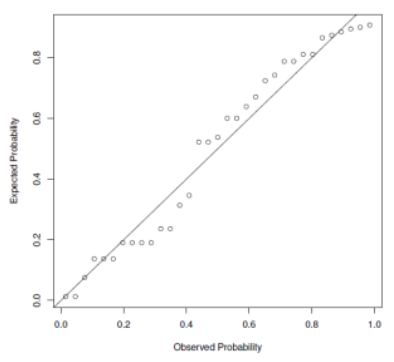

(b) MOBXI

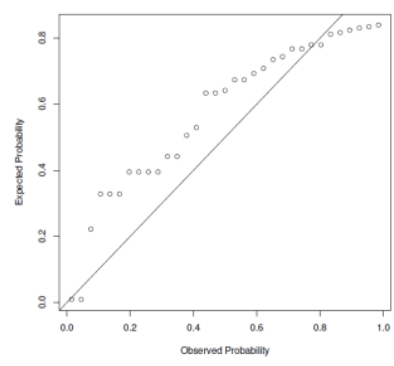

(c) BXII

Figure 2: P-P plots for the Myelogeneous Leukaemia data

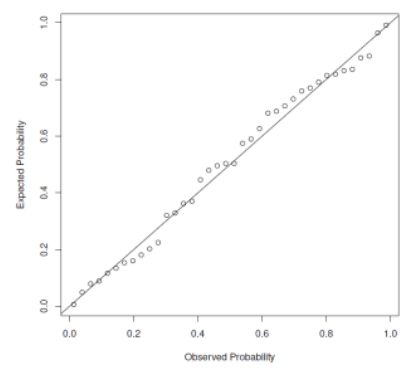

(a) OLBXII

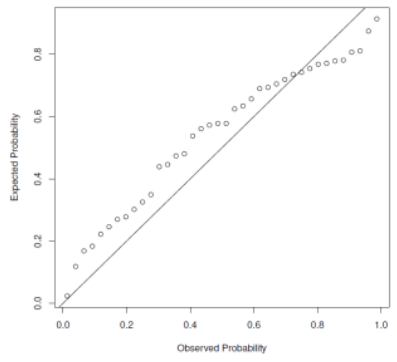

(b) MOBXI

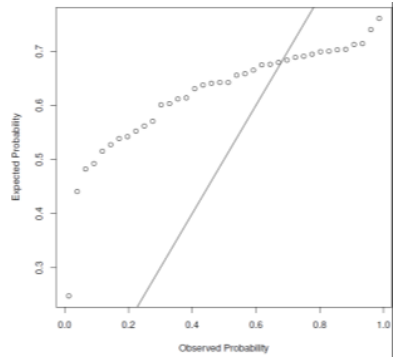

(c) BXII

Figure 3: P-P plots for the Average Annual Percent data 


\subsection{Using Bayesian approach}

In this subsection, a real data set about vinyl chloride data obtained from clean upgradient monitoring wells in $\mathrm{mg} / \mathrm{L}$ that is presented by Bhaumik et al.[4] is used. This data set is 5.1, $1.2,1.3,0.6,0.5,2.4,0.5,1.1,8.0,0.8,0.4,0.6,0.9,0.4,2.0,0.5,5.3,3.2,2.7,2.9,2.5,2.3$, $1.0,0.2,0.1,0.1,1.8,0.9,2.0,4.0,6.8,1.2,0.4,0.2$. The independent priors for this real data are

$$
a \sim \Gamma(0.01,0.01), \alpha \sim \Gamma(0.01,0.01) \text { and } \beta \sim \Gamma(0.01,0.01) .
$$

By using full conditional distributions, a random sample of each parameters with size 700,000 is generated based on Metropolis-Hastings with in Gibbs algorithm. Burning time for removing the effect of the initial values is 10,000 and to avoid correlation problems, a spacing of size 1000 is considered. Thus a sample of size 690 from each parameters is obtained. High autocorrelations within chain indicate slow mixing and slow convergence. The autocorrelation plots for these samples are presented in Fig. 4. We show that the correlation are very small in each sample. The trace plots for these samples are presented in Fig.5. Also, Geweke [12] $\mathbf{Z}$ test is used for testing convergence of each sample to stationary assumption. Geweke method divides each chain into 2 windows containing the first $10 \%$ and the last $50 \%$ of the iterates. If the whole chain is stationary, the means of the values early and late in the sequence should be similar. The convergence diagnostic $\mathbf{Z}$ is the difference between the 2 means divided by the asymptotic standard error of their difference. Thus by considering 5\% for the level test, if the absolute value of $\mathbf{Z}$ is bigger than 0.975 th quantile of normal distribution (1.955), the difference is significant. These values are calculated in Table 5. The histogram of simulated samples from each parameters is shown in Fig. 6. It is worth mentioning that the $\mathbf{Z}$ statistics of this part is obtained by "coda" $\mathrm{R}$ package. In Table 5, we report posterior summaries for the parameters of the OLBXII distribution. Here, SD represents the standard deviation from the posterior distributions of the parameters and HPD represents the 95\% highest posterior density (HPD) intervals.

Table 5: Mean, SD, HPD (95\%) and Z Score for all parameters

\begin{tabular}{ccccc}
\hline Parameter & Mean & SD & HPD (95\%) & Z Score \\
\hline$a$ & 16.450 & 30.780 & $(0.029,81.050)$ & 0.788 \\
\hline$\alpha$ & 1.373 & 0.309 & $(0.783,2.032)$ & 1.918 \\
\hline$\beta$ & 0.306 & 0.338 & $(0.002,0.944)$ & -1.833 \\
\hline
\end{tabular}



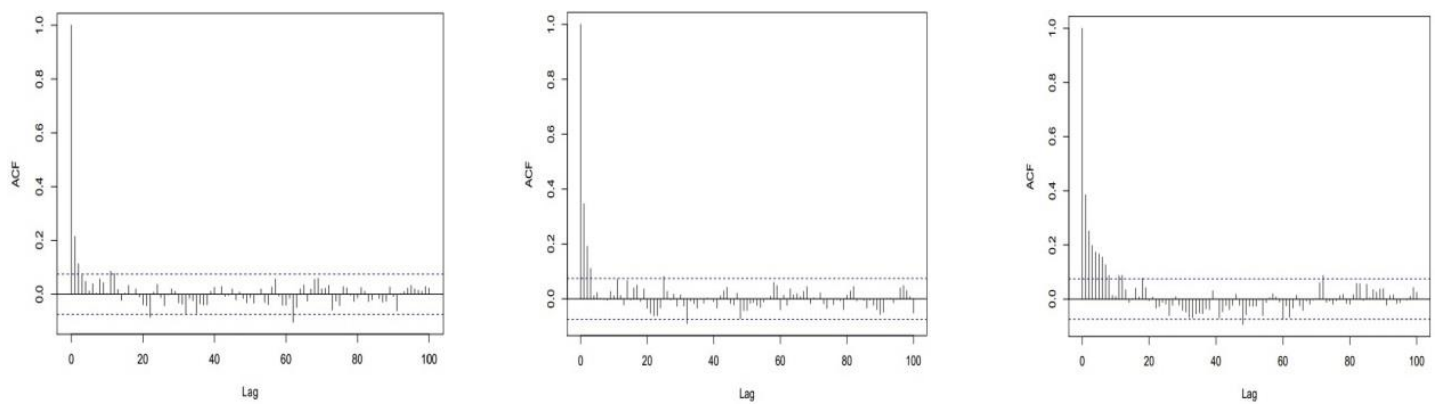

Figure 4: Auto correlation function for parameters: $a, \alpha$ and $\beta$
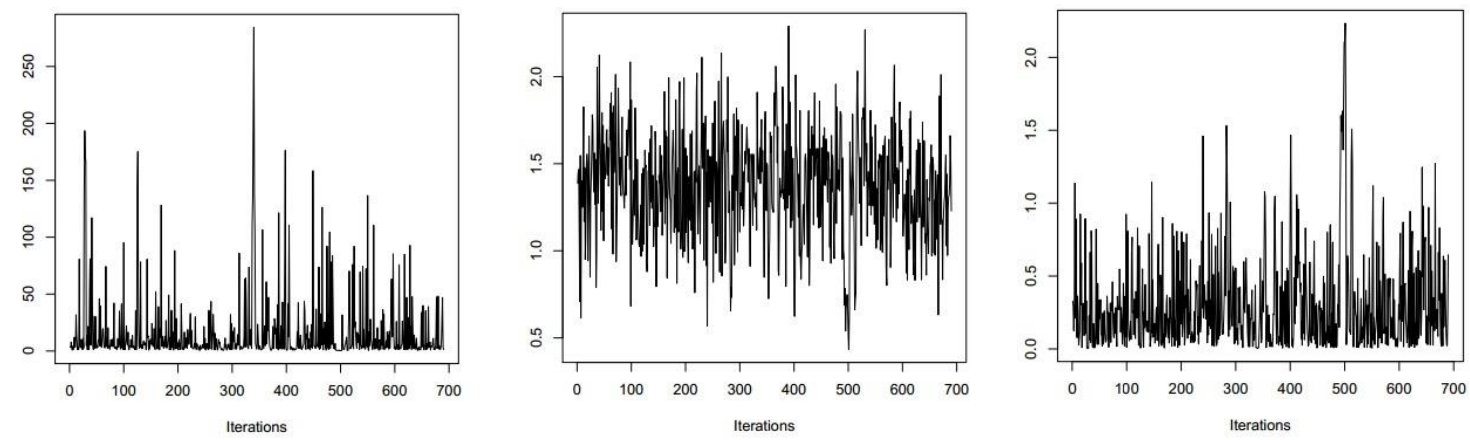

Figure 5: Trace plot for sample of posterior distributions of parameters: $a, \alpha$ and $\beta$. 


\section{Conclusions}

In this article, we study the Odd Lindley Burr XII (OLBXII) distribution which extends the Burr XII (BXII) model. We derive explicit expressions for some of its statistical and mathematical quantities including the ordinary moments, generating function, incomplete moments and order statistics. Some useful characterizations are presented. Maximum likelihood method is used to estimate the model parameters. Simulation results to assess the performance of the maximum likelihood estimators are discussed in case of uncensored data. We demonstrate empirically

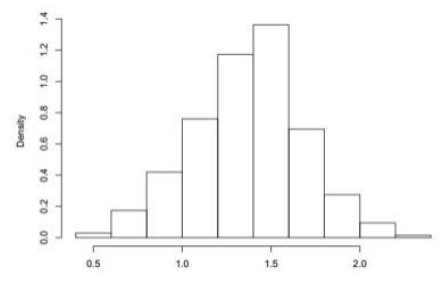

$\alpha$

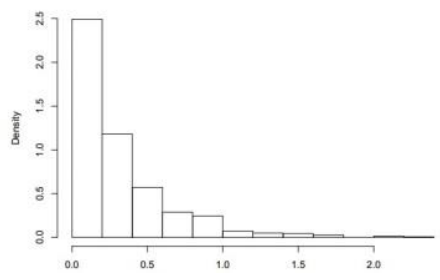

$\beta$

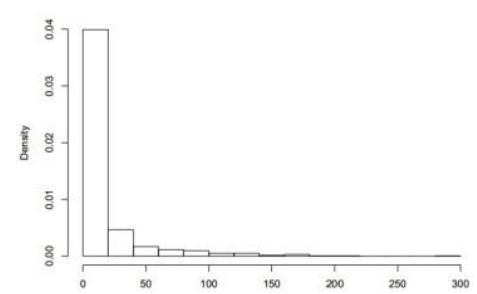

$a$

Figure 6: Histogram plot for sample of posterior distributions of parameters: $a, \alpha$ and $\beta$

the importance and flexibility of the new model in modeling various types of real data sets. Bayesian estimation is performed by obtaining the posterior marginal distributions of model parameters. We use the simulation method of MCMC by the Metropolis-Hastings alogrithm in each step of Gibbs algorithm. The trace plots and estimated conditional posterior models of all parameters are introduced. We hope that the proposed model will attract wider applications in the areas such as engineering, economics (income inequality), survival and lifetime data, hydrology, meteorology and others. 


\section{References}

[1] Afify, A. Z., Cordeiro, G. M., Ortega, E. M. M. Yousof, H. M. and Butt, N. S. "The four- parameter Burr XII distribution: properties, regression model and applications", Commu- nications in Statistics Theory and Method, forthcoming.

[2] Al-Saiari, A. Y., Baharith, L. A. and Mousa, S. A. "Marshall-Olkin extended Burr type XII distribution", International Journal of Statistics and Probability, Vol. 3, No. 1, 2014, pp.78-84,

[3] Altun, E., Yousof, H. M. and Hamedani G. G. (2018). A New Log-location Regression Model with Influence Diagnostics and Residual Analysis. International Journal of Applied Mathematics and Statistics, forthcoming.

[4] Bhaumik, D. K., Kapur, K. and Gibbons, R. D. "Testing Parameters of a Gamma Distribution for Small Samples", Technometrics, Vol. 51, No. 3, 2009, pp. 326-334, www.jstor.org/stable/40586626.

[5] Burr, I. W. "Cumulative Frequency Functions." The Annals of Mathematical Statistics, Vol. 13, No. 2, 1942, pp. 215-232,

[6] Burr, I. W. "Parameters for a general system of distributions to match a grid of $\alpha_{3}$ and $\alpha_{4} "$, Communications in Statistics, Vol. 2, No. 1, 1973, pp. 1-21,

[7] Burr, I. W. and Peter J. Cislak, "On a General System of Distributions: I. Its Curve-Shape Characteristics; II. The Sample Median,” Journal of the American Statistical Association, Vol. 63, No. 322, 1968, pp. 627-635

[8] Cordeiro, G. M., Yousof, H. M., Ramires, T. G. and Ortega, E. M. M. (2018). The Burr XII system of densities: properties, regression model and applications. Journal of Statistical Computation and Simulation, Vol. 88, No. 3, 432-456. 
[9] Elderton, W. P. "Frequency Curves and Correlation", Washington, Harren, 4th Edition, 1953.

[10] Elderton, W. P. and Johnson, N. L., "Frequency Curves and Correlation”, Cambridge University Press, 1969.

[11] Feigl, P., and Zellen, M. "Estimation of Exponential Survival Probabilities with Concomi- tant Information", Biometrics, Vol. 21, No. 4, 1965, pp. 826-838

[12] Geweke, J. " Evaluating the accuracy of sampling-based approaches to calculating posterior moments”, Bayesian Statistics 4 edition, Clarendon Press, Oxford, UK, 1992.

[13] Glanzel, W. "Characterization theorem based on truncated moments and its application to some distribution families," Mathematical Statistics and Probability Theory (Bad Tatz- mannsdorf, 1986), Vol. B, Reidel, Dordrecht, 1987, pp. 75-84.

[14] Glanzel, W., "Some consequences of a characterization theorem based on truncated mo- ments", Statistics: A Journal of Theoretical and Applied Statistics, Vol. 21, No. $\quad$ 4, 1990, pp. 613-618

[15] Gradshteyn, I. S. and Ryzhik, I. M., "Table of Integrals, Series and Products ”, San Diego: Academic Press, 2000.

[16] Hatke, M. A. "A Certain Cumulative Probability Function." The Annals of Mathematical Statistics, Vol. 20, No. 3, 1949, pp. 461-463

[17] Kibria, B. M. G. and Shakil, M., "A New Five Parameter Burr System of Distributions based on Generalized Pearson Differential Equation," Section on Physical and Engineering Sciences - JSM 2011, 866-880. 
[18] Korkmaz, M. Ç. and Erişoğlu, M. (2014). The Burr XII-Geometric Distribution. Journal of Selcuk University Natural and Applied Science, Vol. 3, No. 4, pp. 75-87.

[19] Mead, M. E., "The beta exponentiated Burr XII distribution," Journal of Statistics: Ad-vances in Theory and Applications, Vol. 12, No. 2, 2014, 53-73.

[20] Nasir, M. A. Aljarrah, M,Jamal, F and Tahir, M. H. "A new generalized Burr family of distributions based on quantile function," Journal of Statistics Applications and Probability, 6(3), 2017, pp. 1-14.

[21] Nasir, M. A. Tahir, M. H., Jamal, F. and Ozel, G. "A new generalized Burr family of distributions for the lifetime data," Journal of Statistics Applications and Probability, Vol. 6, No. 2, 2017, pp. 401-417

[22] Paranaiba, P. F. P., Ortega, E. M. M., Cordeiro, G. M. and Pescim, R. R. "The beta Burr XII distribution with application to lifetime data," Computation Statistics and Data Analysis, Vol. 55, No. 2, 2011, pp. 1118-1136

[23] Prudnikov, A. P., Brychkov, Y. A. and Marichev, O. I. "Integrals and Series ", Gordon and Breach Science Publishers, Amsterdam, 1986.

[24] Prudnikov, A. P., Brychkov, Y. A. and Marichev, O. I. "Integrals and Series ”, Gordon and Breach Science Publishers, Amsterdam, 1992.

[25] Ramos, M. W. A., Percontini, A., Cordeiro, G.M. and da Silva, R. V., "The Burr XII negative binomial distribution with applications to lifetime data", International Journal of Statistics and Probability, Vol. 4, No. 1, 2015, pp. 109-125.

[26] Rodriguez, R. N. "A Guide to the Burr Type XII Distributions", Biometrika, vol. 64, no.1, 1977, pp. 129-134, 
[27] Shao, Q. "Notes on maximum likelihood estimation for the three-parameter Burr XII dis- tribution", Computational Statistics and Data Analysis, Vol. 45, No.3, 2004, pp. 675-687,

[28] Shao, Q. Wong, H. and Xia, J. "Models for extremes using the extended three pa- rameter Burr XII system with application to flood frequency analysis" Hydrologi- cal Sciences Journal des Sciences Hydrologiques,Vol. 49, No. 4, 2009, pp. 685-702, http://dx.doi.org/10.1623/hysj.49.4.685.54425.

[29] Silva, F. S., Percontini, A., de Brito, E., Ramos, M. W., Venancio, R. and Cordeiro, G. M. The Odd Lindley-G Family of Distributions. Austrian Journal of Statistics, Vol. 46, No. 1, 2017, pp. 65-87.

[30] Silva, G. O., Ortega, E. M. M., Garibay, V. C. and Barreto, M. L. "Log-Burr XII regression models with censored data", Computational Statistics and Data Analysis, Vol. 52, No. 7, 2008, pp. 3820-3842.

[31] Silva, G. O., Ortega, E. M. M. and Paula, G.A. ”Residuals for log-Burr XII regression models in survival analysis", Journal of Applied Statistics, Vol. 38, No. 7, 2010, pp. 14351445 .

[32] Soliman, A. A. "Estimation of parameters of life from progressively censored data using Burr-XII Model”, IEEE Transactions on Reliability, Vol. 54, No.1, 2005, pp. 34-42,

[33] Tadikamalla, P. R. "A Look at the Burr and Related Distributions", International Statistical Review / Revue Internationale De Statistique, Vol. 48, No. 3, 1980, pp. 337-344,

[34] Wu, S. J., Chen, Y. J. and Chang, C.T. "Statistical inference based on progressively cen- sored samples with random removals from the Burr type XII distribution", Journal of Statistical Computation and Simulation, Vol. 77, No. 1, 2007, pp. 19-27, 
[35] Yousof, H. M., Altun, E., Chakraborty, S. and Handique, L. (2018). Zografos-Balakrishnan Burr XII distribution: regression modeling and applications, International Journal of Math- ematics and Statistics, forthcoming.

[36] Yousof, H. M., Altun, E., Ramires, T. G., Alizadeh, M. and Rasekhi, M. (2018). A new fam- ily of distributions with properties, regression models and applications, Journal of Statistics and Management Systems, Vol. 21, No. 1, 163-188.

[37] Yousof, H. M., Rasekhi, M., Altun, E., Alizadeh, M. Hamedani G. G. and Ali M. M. (2018). A new lifetime model with Bayesian estimation, characterizations and applications. Communications in Statistics - Simulation and Computation, forthcoming. DOI: 10.1080/03610918.2017.1377241

[38] Zimmer, W. J., Keats, J. B. and Wang, F. K. ”The Burr XII distribution in reliability analysis". Journal of Quality Technology, Vol. 30, No. 4, 1998, pp. 386-394.

\section{Appendix A}

Theorem 1. Let $(\Omega, \mathcal{F}, \boldsymbol{P})$ be a given probability space and let $H=[d, e]$ be an interval for some $d<e(d=-\infty, e=\infty$ might as well be allowed) . Let $X: \Omega \rightarrow \mathrm{H}$ be a continuous random variable with the distribution function $F$ and let $q_{1}$ and $q_{2}$ be two real functions defined on $H$ such that

$$
\mathrm{E}\left[q_{2}(X) \mid X \geq x\right]=\mathrm{E}\left[q_{1}(X) \mid X \geq x\right] \eta(x), x \in H,
$$

is defined with some real function $\eta$. Assume that $q_{1} q_{2} \in C^{1}(H), \eta \in C^{2}(H)$ and $F$ is twice continuously differentiable and strictly monotone function on the set $H$. Finally, assume that the equation $\eta q_{1}=q_{2}$ has no real solution in the interior of $H$. Then $F$ is uniquely determined by the functions $q_{1}, q_{2}$ and $\eta$, particularly

$$
F(x)=\int_{a}^{x} C\left|\frac{\eta^{\prime}(u)}{\eta(u) q_{1}(u)-q_{2}(u)}\right| \exp (-s(u)) d u,
$$

where the function $s$ is a solution of the differential equation $s^{\prime}=\frac{\eta \prime q_{1}}{\eta q_{1}-q_{2}}$ and $C$ is the normalization constant, such that $\int_{H} d F=1$. 


\section{Appendix B}

$$
\begin{gathered}
\frac{\partial \ell}{\partial a}=\frac{2 n}{a}-\frac{n}{(1+a)}-\sum_{i=1}^{n}\left[\left(1+x_{i}^{\alpha}\right)^{\beta}-1\right] \\
\frac{\partial \ell}{\partial \alpha}=\frac{n}{\alpha}+\sum_{i=1}^{n} \log \left(1+x_{i}^{\alpha}\right)+(2 \beta-1) \sum_{i=1}^{n} \frac{x_{i}^{\alpha} \log x_{i}}{\left(1+x_{i}^{\alpha}\right)}-a \beta \sum_{i=1}^{n} \frac{x_{i}^{\alpha} \log x_{i}}{\left(1+x_{i}^{\alpha}\right)^{1-\beta}}
\end{gathered}
$$

and

$$
\frac{\partial \ell}{\partial \beta}=\frac{n}{\beta}+2 \sum_{i=1}^{n} \log \left(1+x_{i}^{\alpha}\right)-a \sum_{i=1}^{n} \frac{\log \left(1+x_{i}^{\alpha}\right)}{\left(1+x_{i}^{\alpha}\right)^{-\beta}} .
$$

